\begin{tabular}{|l|l|}
\hline Journal of & \\
Radiotherapy & Journal of Radiotherapy in Practice 2005 \\
in Practice & $4,179-187$ \\
& OCambridge University Press 2005 \\
\hline
\end{tabular}

\title{
Abstracts
}

\section{Abstracts for papers presented at Radiotherapy in Practice 2}

\section{Saturday 17th September 2005}

\section{ADVANCED PRACTICE IN RADIATION THERAPY - THE US PERSPECTIVE}

\section{Dawn Fucillo}

American Society of Radiologic

Technologists, 15000 Central Avenue SE, Albuquerque, NM 87123-3917, USA

This lecture will compare the UK and USA radiation therapy delivery models. This comparison will demonstrate how the UK advance practice model for therapy radiographers may not be applicable in the framework of the USA healthcare system. A brief overview of the development of the Radiologist Assistant will be provided to demonstrate the process for development of a successful advance practice model in the USA. A potential advanced practice model for the USA will be presented and discussed.

\section{SERVICE IMPROVEMENT - WHAT NOW?}

\section{Angie Craig, Peter Kirkbride}

Cancer Services Collaborative 'Improvement Partnership', GF12, Block 4, Mountjoy

Research Centre, Stockton Road, Durham DH1 3UZ, UK

The Cancer Service Collaborative has been running service improvement initiatives and projects since late 1999, with the Radiotherapy team existing for the last 3 years.

This presentation will give an overview of the essence of service improvement, it's practical application and why now, more than ever, it needs to be an essential part of managing and developing modern radiotherapy services - from the viewpoint of a service manager and a Clinical Oncologist who happen to be national project leaders.
With the Cancer Waiting times 31 and 62 day targets, HR Gs, payment by results, patient choice, PACs and NPfIT agendas all descending on us together with some of the biggest workforce changes the NHS has undergone coming to fruition, don't you want some hints and tips on how to cope?

For further information: www.modern.nhs.uk/ radiotherapy or contact the team - 01913868717 . Angie.craig@npat.nhs.uk; Peter.kirkbride@sth. nhs.uk;Trevor.roberts@nuth.nhs.uk

\section{IR(ME)R 2000 IN RADIOTHERAPY - THERE ARE NO PROBLEMS ONLY SOLUTIONS \\ Carol Nix \\ Radiation Protection Division, Health \\ Protection Agency}

The Ionising Radiation (Medical Exposure) Regulations 2000 [IR(ME)R 2000] came into force on 13th May 2000; with the exception of Regulation 4(1) and 4(2) which required the employer to have in place a number of written procedures and protocols. Documentation required for compliance with Regulations 4(1) and 4(2) were to be in place by January 2001.

Five years on there are still elements of the Regulations that cause debate and consternation amongst Radiotherapy professionals.

Amongst these are:

- the entitlement of referrers for radiotherapy,

- who should be entitled to act as a practitioner,

- the difference between professional responsibility and legal responsibility under IR(ME)R 2000,

- delegation of tasks and retention of responsibility,

- what constitutes a "dose much greater than intended",

- how does this fit with a "quality system". 
Staff of IMEeX (Intentional Medical and Environmental Exposure); Radiation Protection Division of the Health Protection Agency have previously had the opportunity to discuss these and other issues at length with staff working clinically. They have also had sight of many procedures, both good and bad. In their new advisory role they are now able to offer suggestions on how to address these issues. The author will look briefly at these issues, demonstrate examples of written procedures and give information on resources where further guidance is available.

\section{THE ADVANCED PRACTITIONER IN NEURO-ONCOLOGY: IMPACT ON SERVICE DELIVERY}

\section{Kate Burton}

Cambridge University Hospitals NHS

Foundation, Oncology Centre, Addenbrooke's

Hospital, Hills Road, Cambridge CB2

2QQ, UK

\section{Background}

The position of specialist radiographer for NeuroOncology was implemented as a result of the increasing complexity of the radiotherapy pathway for these patients. Coordination and interdepartmental liaison was essential to ensure a seamless journey through planning and onto treatment.

Role extension and the inclusion of site-specific radiographers within multi-disciplinary teams were supported by a number of governmental papers including Calman-Hine Report and NHS Cancer Plan. Additionally the major stakeholders in cancer treatment recognised the need to review traditional roles, to develop new roles that crossed professional boundaries and to widen opportunities for professional development.

\section{Impact of the role}

Since its introduction the role has developed and advanced in many directions. Whilst the need to ensure a smooth, trouble-free radiotherapy pathway remains a priority, this is balanced with the need to provide holistic, patient-centred care. Knowledge and training to support and develop the role has been provided by work-based training units and M-level modules.
The advanced practitioner for NeuroOncology is the key worker for patients during their journey through radiotherapy. The position offers a consistent point of contact, providing expert information, support and care and includes performing radiographer-led review clinics. A radiographer-run service for palliative radiotherapy has improved service delivery both in terms of patient support and departmental throughput. The role has facilitated the introduction of complex radiotherapy techniques aimed at improving patient outcome in terms of toxicity and prognosis.

\section{Conclusion}

This role has promoted patient-centred care, improved technical radiotherapy delivery and facilitated research within a specialist NeuroOncology centre.

\section{RADIOTHERAPY REVIEW AND ASSESSMENT \\ Pat McCabe, Margaret McLean \\ Department of Radiotherapy and Oncology, James Cook University Hospital, Marton Road, Middlesbrough, UK}

For several years we have worked in the oncology clinics providing information on chemotherapy, radiotherapy and clinical trials. Working across the Cancer Alliance we provide continuity of care and holistic assessment for patients in both the peripheral hospital clinics and the cancer centre. Following patients throughout their cancer journey we provide supportive care from diagnosis, recurrence, to palliative care. As multidisciplinary working evolved we became active members of the team.

From this we were invited by the consultants to undertake treatment reviews and commenced the MSc assessment and review module. This module helped us to critically assess the advice given and the medications prescribed. Through our study we recognised our practice was ritualistic and there was a need to develop evidence based protocols for specific treatment sites.

Working in the radiotherapy review clinic and follow up clinics it became apparent that patients 
were being discharged from our care when their treatment reaction was at its peak. This prompted the development of the first therapist led clinic with a dedicated appointment with the review radiographer and a dietician. This is proving very effective and we believe this is multidisciplinary working at its best in providing best practice and holistic care.

The advantages of the role are significant with improvement in patient care by providing holistic assessment, continuity of care and evidence based practice. As a result of our role, the profile of therapy radiographers has been raised within the Cancer Alliance.

\section{RADIOGRAPHER LED BREAST SIMULATION - SETTING UP THE SERVICE AND REAPING THE REWARDS}

\section{Jan Johnson}

Sheffield Teaching Hospitals Foundation
Trust, Weston Park Hospital, Whitham
Road, Sheffield S35 7DE, UK

Developing and implementing a radiographer led breast simulation service requires a thorough assessment of local departmental needs; there is no scope for a "one size fits all" approach. Each department should design its radiographer led service around a thorough assessment of clinical need, analysing skills inherent amongst it's radiographers, accessing high quality training, establishing co-operation and mutual respect within the multi-disciplinary team and engendering a culture of constant monitoring and evaluation.

However, once implemented, the radiographer led breast simulation service can potentially offer manifold benefits for the patient, the radiographers and the service as a whole. Skill mix and advancing roles can bring greater flexibility to reduce clinic and national waiting times as well as enhancing the continuity experienced by the patient.

With radiographers embarking on this advanced role there becomes further scope for the development of specialist services to patients receiving breast radiotherapy. Radiographers may build on the foundation of breast simulation work by extending their scope of practice into consenting, image evaluation, assessing first day set ups and various planning roles, possibly leading to a redefining of specialist and advanced roles.

Our experience in Sheffield of undertaking radiographer led breast simulation has been both positive and successful. A small group of radiographers undertake both conventional and virtual simulation procedures and offer a degree of flexibility which complements, not detracts from the consultant led sessions.

\section{ADVANCING RADIOGRAPHER PRACTICE IN GYNAECOLOGICAL ONCOLOGY \\ Lisa Punt \\ Addenbrooke's NHS Trust Box 193 , Addenbrooke's Hospital, Hills Road, Cambridge CB2 2QQ, UK}

\section{Background}

Following publication of the Calman-Hine report in $1995^{1}$ a revolution in cancer care was about to ensue. The report highlighted key principles that were to govern the future provision of cancer care. In order to meet these principles a restructuring of cancer services was outlined. The report suggested a new 3-tier structure consisting of primary health care, cancer units and specialist cancer centres. It was suggested that a multi-professional site specific approach would facilitate an integrated specialist service providing uniformly high quality care to all patients. Gynaecology was one of the five original site-specific groups identified within the report, targeted to undergo radical change.

In 2000 the government published 2 reports The NHS Plan ${ }^{2}$ and Meeting the Challenge ${ }^{3}$ both of which outlined modernisation and restructuring of the NHS. The reports identified new ways of skill mixing and role extension of health care professionals to facilitate improved service quality and better patient outcomes. It was also suggested that the new structure would allow staff to be recognised for the role they undertook, ultimately improving recruitment and retention. 
It is the impact of both restructuring of service delivery and changes in professional development that has been the impetus behind advancing practice for the radiographer and improving service quality for the patient.

Aims

To discuss how service development was identified and implemented within the Authors Gynaecological Oncology Centre. Considering the educational and training requirements. Identifying areas of role extension and the impact this has had on the Centre and the patient. Also to consider the constraints of such a role and how these have been addressed. Finally hypothesise development of the role and the individual.

1 The Calman-Hine report. www.dh.gov.uk 1995

2 The NHS Plan: A plan for investment, a plan for reform. www.dh.gov.uk 2000

3 Meeting the Challenge:A strategy for the Allied Health Professionals. www.dh.gov.uk 2000

\section{"FIVE FOR THE ROAD" - AN UPDATE ON THE ONTARIO- BASED PROJECT EXPLORING ADVANCED PRACTICE FOR RADIATION THERAPY}

\section{N. Harnett, A. Bolderston}

Princess Margaret Hospital, University Health Network, 610 University Avenue, Toronto, Ontario, Canada M5G 2 M9

Ontario, like other Canadian provinces and countries around the world, has traditionally had to deal with the recurring shortages of radiation medicine professional by forcing patients to wait for access to treatment, sending patients for treatment to distant facilities who could accommodate an additional workload and by undertaking aggressive and expensive recruitment schemes. While temporarily successful, it seemed that long-term solutions were never realized. In an effort to find a permanent solution to this issue, the Ministry of Health and Long Term Care (MOHLTC) in Ontario expressed its commitment to creating a more "flexible" radiation medicine workforce - one that would be better able to respond to temporary fluctuations in staffing in any of the three disciplines involved. To that end, the MOHLTC has funded the "Advanced
Practice for Radiation Therapy" project to help examine a variety of creative, non-traditional roles for radiation therapists within radiation treatment services. Five pilot projects are currently underway exploring the nature of roles that would best serve this purpose. Each site implementing a pilot project have been charged with creating a final competency profile, collecting data on the service improvements that would be recognized, and identifying the educational needs of the roles. This presentation will highlight the advanced roles being explored including the service gaps they propose to fill, and the data being collected to assess outcomes and inform the next steps of this initiative. An initial review of licensure issues for the implementation of an "advanced practice" class within the province of Ontario will also be discussed.

\section{INTEGRATING ACADEMIC AND CLINICAL ROLES FOR RADIATION THERAPISTS: THE PRINCESS MARGARET HOSPITAL EXPERIENCE}

\section{Amanda Bolderston, Pamela Catton, Julie Wenz, Mary Gospodarowicz}

Princess Margaret Hospital, University Health Network, 610 University Avenue, Toronto, Ontario, Canada M5G 2 M9

Toronto's Princess Margaret Hospital is the premier radiation treatment centre in Canada and one of the largest comprehensive cancer treatment and research facilities in the world. To profit from the intellectual capital of all radiation professional groups in advancing the academic and clinical programs, the $\mathrm{PMH}$ Radiation Medicine Program recently proposed a new model of Advanced Integrated Practice (AIP) for radiation therapists. The AIP model is modelled on academic physician practice with its combined roles for the expert clinician specialist as well as responsibilities in roles such as scientist, educator, clinical investigator, leader or manager.

Goals for the AIP model for therapists were:

1. Growth of expertize in treatment planning and delivery.

2. Opportunity for clinical academic careers in radiation therapy in the areas of clinical and translational research and education. 
3. Opportunity to acquire and practice leadership and management skills.

4. Opportunity to combine radiotherapy practice with informatics.

5. Opportunity to combine practice and graduate studies.

A number of specialized positions have been created to support this model, including five integrated clinical-research roles; several "practice development" positions and specialized educational roles. This presentation will include an overview of AIP and will discuss the preliminary assessment of the promises and challenges of the model, namely:

1. Qualitative and quantitative data from surveys, discussions and individual interviews.

2. A review of patient satisfaction data.

3. An initial process review of implemented job models, career opportunities, research involvement and academic output.

The presentation will also share the projected future directions of the model.

\section{DEVELOPING TECHNOLOGIES AND THE ADVANCING ROLE OF THE THERAPY RADIOGRAPHER}

\section{McCarthy, J. Davies, J. Stratford, M. Duffy}

Wade Centre for Radiotherapy Research, Christie Hospital, Wilmslow Road, Withington, Manchester M20 4BX, UK

The last decade has seen a rapid development in the technology that is available to us. This has led to the development of more complex therapies such as intensity modulated treatments. These complex therapies have highlighted the need for integrated on-treatment imaging devices. The conception and introduction of equipment such as X-ray volumetric imaging (XVI) will culminate in the ability to not only examine bony displacements, but will allow PTV verification, assessment of tumour margins as well as tumour tracking. These changes will facilitate the development of image guided and adaptive radiotherapy. The experience and ability of therapy radiographers is paramount to improving and advancing this sphere of radiotherapy practice and development.
The implementation of XVI and adaptive therapy can only be initiated within a clinical setting if therapy radiographers are making instant, autonomous on-line decisions. These issues highlight the need to adopt a solid multi-disciplinary approach to ontreatment verification whilst maximising skill-mix and extending the role of the therapy radiographer. The assessment and delineation of the PTV as well as organs at risk and the implementation of a "plan of the day" system, incorporating fluoroscopic tumour tracking and the calculation of dose delivered will become standard practice. The development of skills is fundamental to advancing practice. Additional training, competency assessment and the setting of national standards to ensure proficiency and safety are all crucial.

\section{RADIOTHERAPY STAFFING MODELS AND FOUR TIER ESTABLISHMENTS}

\section{S. E. Griffiths}

Cookridge Hospital, Leeds Teaching Hospitals NHS Trust, Leeds LS16 6QB, UK

Ultimately an overall multidisciplinary staffing levels guidance for radiotherapy is needed, inclusive of four tier and skill mix guidelines. In the interim the Institute of Physics and Engineering in Medicine (IPEM) and the Royal College of Radiologists both have publications detailing their staffing requirements for radiotherapy services. The IPEM framework seems particularly useful to adapt for radiographers for whom no such guidance currently exists.

A survey of staff and services within the budgetary control of radiotherapy service managers was collected from 43 UK centres for 2003/4 and used to calculate the average number of WTE staff (radiographers and support staff) required to run radiotherapy services. The measure adopted was WTE per linac hour of service which can be related to activity and demand, for treatment and simulation services inclusive of associated management and training functions. A model is suggested for additional staff delivering services such as dose planning, mould room etc. based on WTE per million population served, likely to have a multi-disciplinary makeup. 
Suggested skill mix models for the four tiers can be applied to the WTE framework to inform the makeup of establishments and the expected career structure.

\section{IMMERSIVE VISUALIZATION ENVIRONMENTS FOR PRACTICAL SKILLS TRAINING IN RADIOTHERAPY}

\section{Rob Appleyard, Pete Bridge,} Prof. Andy Beavis

Faculty of Health and Wellbeing, Sheffield Hallam University, Prof. Roger Philips and James Ward - Department of Computer Science, Hull University

Introduction and purpose

It is hoped that an "immersive" visualization environment (IVE) providing an accurate, interactive simulation a virtual treatment room will enable radiotherapy students to gain valuable and realistic experience that supplements their clinical education without burdening departments.

Spatial ability as a predictor of clinical set-up ability has been previously highlighted ${ }^{1}$ and although no significant correlation between spatial ability was found this may have been due to sampling inadequacies. We aim to address these.

The purpose of our work is to evaluate the potential of using an IVE for practical skills training for pre-registration radiotherapy students. It has 2 main aims:

- to assess student perceptions of using an IVE for the development of essential practical skills in a non-clinical environment;

- to determine any correlation between spatial ability and clinical set-up ability using the IVE in order to assess whether its use may be targeted for certain individuals.

\section{Method}

A skin apposition simulator (SAS) that replicates a working linear accelerator (and controlled using a genuine hand pendant) utilizes the $16 \times 8$ foot stereoscopic work wall in the Hull Immersive Visualization Environment. Patient data was created using the Virtual Human dataset with treatment sites drawn on. Simple algorithms objectively assess accuracy of clinical set-up.

Sixty first-year students evaluated the application with quantitative and qualitative data collected using pre and post questionnaires. Students were provided with an opportunity to experience the SAS in groups and individually. Each student was also required to perform a specific clinical setup that was objectively assessed and timed. The results of this were correlated with spatial ability assessed using the lego block test.

Results and conclusions

Results and conclusions are not yet available but will be presented.

Contents of presentation

1. Rationale for using IVE's.

2. Brief description of the application (further details on poster).

3. Results.

4. Conclusions and thoughts for further work.

\section{Sunday 18th September 2005}

\section{TOMOTHERAPY - FROM CONCEPT TO CLINICAL REALITY}

\section{Thomas Rockwell Mackie}

University of Wisconsin, 1300 University Avenue, Madison, Wisconsin, WI 53706 , USA

Helical tomotherapy is an intensity-modulated radiotherapy (IMRT) system placed into the ring gantry of a helical CT scanner. An intensitymodulated fan beam rotates continuously as the couch translates through the large-bore CT-like gantry. Megavoltage computer tomographic (MVCT) images are acquired using a conventional CT detector and data acquisition system.

Tomographic images are the basis for novel radiotherapy verification processes. The MVCT scans can be acquired just before treatment and fused at the treatment console with diagnostic CT scans to determine if the patient has been properly setup. We have begun clinical evaluation of the system. MVCT scans human patients will be 
presented. The MVCT images, at patient doses comparable to those from a conventional diagnostic CT scanner, can for example reveal the presence of lung tumours and the location of the prostate. The MVCT scans were used to evaluate the accuracy of setup for IMRT.

We anticipate that having CT images at the time of treatment will enable new quality assurance and corrective procedures that we collectively call adaptive radiotherapy that will allow patient-specific quality assurance for the entire course of treatment.

\section{DOES A PRE-RADIOTHERAPY INFORMATION APPOINTMENT HAVE THE EFFECT OF REDUCING ANXIETY IN PATIENTS WITH CANCER UNDERGOING A COURSE OF RADIOTHERAPY TREATMENT?}

\section{Samantha Bostock}

Gloucestershire Oncology Centre, Cheltenham General Hospital, Sandford

Road, Cheltenham, Gloucestershire

GL53 7 AN, UK

\section{Aim}

To investigate the effectiveness of a pre-treatment individualised information intervention predicted to improve cancer patients' experience of radiotherapy treatment by reducing anxiety using the professional expertise of the therapeutic radiographer.

\section{Introduction}

This research builds on current recommendations that measures be taken at a local level to ensure cancer patients receive high quality information. There is no research that tests such a focussed information and support intervention by therapeutic radiographers.

Methodology

The intention of identifying a cause-effect relationship between the pre-radiotherapy appointment and a reduction in the score on an anxiety scale led to a positivist approach and a study conducted within the empirico-analytical paradigm.

\section{Method}

A between-groups randomised control trial. One experimental and one control group.

\section{Participants}

Seven female and three male participants over the age of thirty-five with a range of cancer types.

\section{Measure}

Spielbergers' (1983) State Trait Anxiety Inventory (STAI) was administered before and after the intervention.

\section{Results}

Anxiety scores were analysed using the MannWhitney U Test due to small numbers of participants. A trend $(p=0.095)$ towards a decrease in state anxiety for the experimental group and an increase in state anxiety for the control group prior to the first radiotherapy treatment was shown.

\section{Conclusion}

The results corroborate other research, which found radiotherapy to be an anxiety-provoking event, and support the theoretical basis of why information helps.

\section{Recommendations}

For research - to investigate if high initial anxiety is indicative of responsiveness to information, to explore the timing of the intervention in relation to pre-treatment appointments and treatment, to continue the study to recruit adequate numbers of participants. For practice - to consider an extra appointment for patients in order to deliver the information intervention.

\section{PROSTATE IRRADIATION IN PATIENTS WITH HIP PROSTHESES}

\section{Laura James}

North West Medical Physics, Christie Hospital, Wilmslow Road, Withington, Manchester, UK

Aims

To identify if an optimum beam arrangement exists to enable accurate delivery of radiotherapy to patients with a prosthetic hip. 
Methodology

Treatment options are avoidance, compensation or IMRT. Each of these options was examined. Avoidance was chosen for further investigation. Ten previously planned "normal" patients were selected. All had early stage prostate cancer. The hip was outlined and treated as though it was metallic. Several plans were produced for each patient, and dose volume histograms for rectum, bladder and contra-lateral hip were compared.

Results

The three field anterior, posterior and lateral (APL) plans and three field oblique plans gave the lowest rectal doses. The three field APL plans delivered higher bladder doses, the three and four field obliques gave lower bladder doses. The four field diamond showed no significant difference. For the contra-lateral hip, the dose is lowest with four field oblique and diamond plans. On the basis of these results, the author would recommend that either the three field oblique or three field APL plan be used. It gives the lower rectal doses, and the two plans can be compared for their bladder and contra-lateral hip doses.

\section{Conclusions}

When planning radiotherapy for early stage prostate cancer in patients with artificial hip joints, the simplest thing to do is to avoid the prosthesis. The beam arrangements of choice should be three field APL, or three field obliques. Further work should include a feasibility study on the use of IMRT.

\section{AN INVESTIGATION INTO THE PERCEIVED USEFULNESS OF PRECEPTORSHIP. \\ A COMPARATIVE STUDY BETWEEN TWO \\ RADIOTHERAPY CENTRES}

\section{Mandy Harbottle}

University of the West of England, Glenside Campus, Blackberry Hill, Stapleton, Bristol BS16 1DD, UK

Aims

This study explored the perceived usefulness of preceptorship between a department that had implemented a successful preceptorship programme, and a department that hadn't implemented preceptorship.

The intention was that the findings would inform the design and implementation of a preceptorship programme.

\section{Introduction}

The College of Radiographers recommends preceptorship for newly qualified radiographers $[1,2$, 3]. Personal experience highlighted concerns regarding the lack of understanding of preceptorship. Where a preceptorship program had been implemented, the program was beneficial, and could potentially alleviate some of the recruitment and retention issues faced within radiotherapy.

\section{Method}

An exploratory, qualitative study was undertaken within the constructivist paradigm to explore the perceptions of what radiographers understand preceptorship to be, the problems with implementing preceptorship and the potential benefits of preceptorship. Data collection was through a focus group interview with each department taking part that was transcribed and analysed using Burnard's fourteen stage analysis [4].

\section{Findings}

Time, definition and understanding, professionalism and implementation were the major themes that occurred from the interviews. These themes had different meanings for each of the groups.

\section{Conclusions}

Preceptorship can be a useful tool for newly qualified radiographers. Careful consideration needs to be given to disseminating a definition and a framework for its implementation. Having a member of staff who is responsible for coordinating a preceptorship programme would appear to add to the success of implementing a programme in clinical practice.

\section{Recommendations}

The level of knowledge and understanding of preceptorship needs to be explored in detail prior to implementing a preceptorship program. 


\section{References}

1. DoH. Radiography Skills Mix. A report on the four-tier service delivery model. DoH London, 2003.

2. The College of Radiographers, The Society of Radiographers. Clinical Supervision Framework. The College of Radiographers. London, 2003.

3. The College of Radiographers. A strategy for the education and professional development of radiographers. The College of Radiographers. London, 2002.

4. Burnard P. A method of analysing interview transcripts in qualitative research. Nurse Education Today 1991;11: 461-466.

\section{IMPLEMENTATION OF THE FIRST AUSTRALIAN GRADUATE ENTRY MASTERS DEGREE IN RADIATION THERAPY \\ Caroline Wright}

Monash University, Dept Medical Imaging and Radiation Science, Building 13C, Clayton Campus, Wellington Road, Clayton, Victoria 3800, Australia

In July of 2003, the first graduate entry Masters in Radiation Therapy commenced at Monash University. This opened a new pathway to professional accreditation.

The paper presents the experience so far and directions for the future. One of the virtues of a graduate entry program is the fact that graduates enter the course having developed many of the "thinking and learning" skills needed to accelerate both academic and clinical development. The "flexible learning" mode of delivery of the academic units and the clinical placement schedule attempts to address issues that have previously been seen as challenges to radiation therapy education in Australia. Students throughout Australia are able to access the course and study academic units at home. This removes the need for re-location and also allows students in year 1 to maintain part-time employment. Recruitment of local students allows regional centres to have ownership of clinical training and retention in the workforce post qualification (this is important in states which do not have undergraduate programs feeding recruitment). This is further consolidated in year 2 when the students are paid a training wage for their 48 week professional clinical placement.

Development of the course so far, has been achieved by employing a seamless communication and feedback system, based upon responsiveness to the needs of all stakeholders. The first cohort of students will enter the profession in July 2005 and we are currently designing a longitudinal study from which we can monitor the career pathway of these individuals. 
https://doi.org/10.1017/S146039690528024X Published online by Cambridge University Press 